\title{
Myth busting patient's pain: comparing robotic-assisted verses open radical prostatectomies
}

\author{
Benjamin Condon $^{1,2}$, Dominic Bagguley ${ }^{1,3}$, Nathan Lawrentschuk ${ }^{1,4,5}$ \\ ${ }^{1}$ EJ Whitten Prostate Cancer Research Centre at Epworth, Melbourne, Australia; ${ }^{2}$ Department of Urology, St Vincent's Hospital Melbourne, \\ Melbourne, Australia; ${ }^{3}$ Department of Urology, Northern Health, Melbourne, Australia; ${ }^{4}$ University of Melbourne, Department of Surgery, Austin \\ Hospital, Melbourne, Australia; ${ }^{5}$ Peter MacCallum Cancer Centre, Department of Cancer Surgery, Melbourne, Australia \\ Correspondence to: Benjamin Condon. EJ Whitten Foundation Prostate Cancer Research Centre, Epworth HealthCare, 124 Grey St. Richmond, \\ Victoria 3121, Australia. Email: bencondon34@gmail.com. \\ Provenance and Peer Review: This article was commissioned and reviewed by the Section Editor Dr. Xiao Li, MD (Department of Urology, Jiangsu \\ Cancer Hospital \& Jiangsu Institute of Cancer Research \& Nanjing Medical University Affiliated Cancer Hospital, Nanjing, China). \\ Comment on: Knipper S, Hagedorn M, Sadat-Khonsari M, et al. Comparison of intra- and postoperative analgesia and pain perception in robot- \\ assisted $v s$. open radical prostatectomy. World J Urol 2019. [Epub ahead of print].
}

Submitted Nov 29, 2020. Accepted for publication Jan 02, 2020.

doi: 10.21037 /gs.2020.01.01

View this article at: http://dx.doi.org/10.21037/gs.2020.01.01

\section{Introduction}

Radical prostatectomy (1) is one of the most common treatments for locally advanced prostate cancer $(\mathrm{PCa})$. Robot-assisted radical prostatectomy (RARP) is the predominant surgical approach in many industrialised countries (2). One of the most common purported benefits of RARP over open radical prostatectomy (ORP) is reduced pain and analgesic requirement, however, a scarcity of reliable studies exist to validate this claim $(3,4)$. Intuitively, the more minimally invasive technique can be reasoned to reduce patient's pain both intraoperatively and in the weeks following. Whilst being well-intentioned, this rational is not supported in the literature.

\section{Context \& epidemiology}

Prostate cancer is the second most common cancer diagnosis and the fifth leading cause of death in males globally. In 2018 there were 1,276,106 new registered diagnoses of prostate cancer, representing $7.1 \%$ of cancers in males worldwide (2). Both the incidence and mortality rates in prostate cancer are concordant with advancing age. The average age of prostate cancer diagnosis is 66 years and the incidence rate of prostate cancer in men over the age of 65 approaches $60 \%$. Well known risk factors include age, ethnicity, genes and family history $(3,4)$, while other factors associated with prostate cancer include diet, obesity, sedentary lifestyle, inflammation, hyperglycaemia, infections, and exposure to environmental chemicals or ionizing radiation (2,5-7).

$\mathrm{RP}$, in its various iterations, has been the mainstay of surgical treatment for locally advanced prostate cancer for many years. Since 1990, RARP has provided an alternative approach to both open (retropubic) and laparoscopic RP (LRP). In the almost 20 years since, RARP has been refined and benefited from technological advances, but there remains little prospective data in the literature comparing the efficacy of each approach, both in terms of oncological outcomes as well as assessing technical factors like complication rates and postoperative pain. More recently even more minimally invasive and organ-sparing therapies such as focal therapy have gained traction but lack longterm data (8).

\section{Aim of radical prostatectomy}

Regardless of specific surgical approach, RP's primary aim is to surgically maximise oncological outcomes for each patient, specifically prostate cancer-free survival, overall survival and biochemical recurrence-free survival whilst at the same time maximising urinary and sexual quality of life 
and minimising surgical complications, postoperative pain and length of stay in hospital amongst other metrics.

\section{Article in focus}

A recent study by Knipper et al. (9) brings us a prospective comparison of intra- and postoperative analgesia and pain perception in patients undergoing RARPs versus ORPs in a 551 consecutive patient cohort, from a single centre. Four experienced surgeons performed both operations with a standard intra and post-operative pain regime that was recorded on an electronic record of administration of analgesic medication (as is mandatory in Germany). The study was aided further by the German reimbursement policy, which meant patients in both groups stayed in hospital for 6-8 days postoperatively, enabling consistent recording of pain and medication use in both cohorts.

Significantly, the results of this study contradict the notion that RARP has reduced pain and analgesic requirement when compared to ORP. There was no significant difference in median dose of morphine equivalent analgesic administered in RARP compared to ORP. Additionally, there was no significant difference in mean maximum perceived pain score postoperatively on day 0 , where ORP patient's scored their pain higher than RARP patients (3.2 vs. 3.6, $\mathrm{P}=0.1$ ). This score decreased within the following days, and again no significant difference between the two was reported. The only significant difference identified between RARP and ORP was the longer operating time associated with RARP (175 vs. $155 \mathrm{~min}$, $\mathrm{P}<0.001)$ and median dose of metamizole intraoperatively, even after being adjusted for length of surgery and patient BMI (146.7 mg in RARP vs. $133.9 \mathrm{mg}$ ORP, $\mathrm{P}<0.001)$ (9).

Whilst these findings may prove to dispel common misconceptions about pain post RARP compared to ORP, there are some limitations to this study which fail to highlight other significant factors that contribute to deciding between the two approaches. Specifically, length of stay being determined by renumeration rather than clinical factors means the length of stay of RARP patients is artificially longer than we typically would see clinically (10). In a broader context, the standardised intra-operative and postoperative analgesic regimen used by Knipper et al. consistently enables a more accurate analysis between groups is a strength of this study. However, there remains no widely used and accepted analgesic framework in the literature, with some studies not disclosing their pain regime, rendering it difficult to compare the results of this study with others previously published.

\section{Literature review}

Knipper et al.'s findings contradict those of Yaxley et al. in 2016 (11) where published early post-operative data (at 12 weeks) from a prospective randomised phase 3 trial compared RARP ( $\mathrm{n}=151)$ and ORP $(\mathrm{n}=157)$ in clinically localised PCa. The study was based at a single centre, and each operative group had the same surgeon perform each procedure. The trial was powered to assess both healthrelated and domain specific quality of life outcomes over 24 months, including postoperative pain using the Surgical Pain Scale (3), Revised Impact of Events (RIES) (12) and Hospital Anxiety and Depression Scale (HADS) (13). Pain was assessed at 24 hours, 1 week, 6 weeks and 12 weeks after surgery. There was no significant difference in pain at rest between the two groups at any time point. For pain during normal activities, participants in the RARP group had significantly less pain in the very early post-operative period ( 24 hours and 1 week) but not at 6 or 12 weeks after surgery. A similar pattern was also noted for worst reported pain measure, with RARP group patients reporting less pain at 24 hours and 1 week, but not at later time points.

At 12 weeks, both operative techniques yielded similar functional outcomes as well as having no significant difference in postoperative complication rate and intraoperative adverse events, as was the case in Knipper et al.'s study. Despite the RARP group reporting higher physical quality of life, and significantly less distress (HADS; RARP $=2.30$, ORP 3.51, $\mathrm{P}=0.03$ ) in the 12 weeks postoperatively, there was no significant difference in return to work time. Interestingly, the length of time taken to perform surgery in the RARP group was significantly less than the ORP group $(\mathrm{P}<0.0001)$ but there was no difference in time in the recovery room $(\mathrm{P}=0.95)(11)$.

However, findings in a 2005 prospective study by Webster et al. (14) were more aligned with that of Knipper et al.'s (9). The total mean morphine sulfate equivalent \pm SD in patients in the RARP and ORP groups was low and, when corrected for length of stay, it was not statistically different $(22.41 \pm 1.13$ vs. $23.01 \pm 1.16 \mathrm{mg}, \mathrm{P}=0.72)$. Mean Likert pain perception scores were low at all time points in the RARP and ORP groups but statistically lower on the day of surgery in the RARP cohort $(2.05 \pm 1.99 v s$. $2.60 \pm 2.25, \mathrm{P}=0.027)$. Patient reported mean pain scores 
were almost identical for RARP $v s$. ORP on postoperative days $1(1.76 \pm 1.87$ vs. $1.73 \pm 1.77, \mathrm{P}=0.880)$ and $14(2.51 \pm 1.91$ vs. $2.42 \pm 1.84, \mathrm{P}=0.722$ ) (14). Perioperative narcotic use and patient reported pain are low regardless of the surgical approach used for RP. RARP did not provide a clinically meaningful decrease in pain compared with (retropubic) ORP, primarily because of the low pain scores reported in each group (14).

Systematic review and meta-analysis by Ilic et al. of all randomised and pseudo-randomised trials published up until July 2017, were analysed to determine the comparative effects of laparoscopic RP (LRP), ORP and RARP in men with localised PCa (1). Postoperative pain comparison was identified as a secondary outcome. Ilic et al. found two papers that supported a small, and insignificant, reduction in post-operative pain from day 0 to day 7 in LRP and RARP patients compared to ORP, however they assessed the quality of this evidence to be low. Importantly, they found no study that addressed the outcomes of prostatecancer specific survival, biochemical recurrence-free survival or overall survival. They also found RARP posing little to no difference in overall surgical complications, again with low quality evidence and little to no evidence of a difference in urinary quality of life and sexual quality of life between RARP, ORP and LRP with moderate quality of evidence (1).

D'Alonzo et al.'s 2009 retrospective comparison of the anaesthetic management of RARP and ORP found reduced blood loss, fewer blood transfusions, reduced intraoperative vasopressor requirement and reduced surgical times for RARP when compared to ORP (10). With respect to analgesic consumption, intraoperative opioid requirement [morphine equivalent $(\mathrm{mg})$ ] was similar between RARP and ORP (mean \pm SD: $52 \pm 24$ vs. $50 \pm 23$ ), as was intraoperative ketorolac use (n) $(52 \pm 20$ vs. $48 \pm 17)$. In post-anaesthetic care unit, opioid requirements were significantly less in RARP vs. ORP $(11.4 \pm 7.7$ vs. $14.9 \pm 9.8 \mathrm{mg} ; \mathrm{P}<0.0001)$ with comparable ketorolac use $(167 \pm 65$ vs. $174 \pm 62)(10)$. From the perspective of comparing analgesic requirement in RARP $v s$. ORP, some limitations of this study include pain scores were not being recorded; the analgesic regime not being standardised; and the study only comparing opioid consumption (and not other analgesic agents) in the immediate post-operative period.

Haese et al.'s 2019 prospective data set of oncological, functional and surgical outcomes of ORP vs. RARP failed to address peri and postoperative pain within their study design (15). Despite demonstrating no difference in 48-month biochemical recurrence, erectile function, and in any markers of surgical outcome (including: rate of nerve-sparing procedures, lymph node yield, surgical margin status, length of hospital stay, operation time, blood loss, transfusion rate, time to catheter removal), their data set is of little value when considering relative analgesic requirements and comparative pain.

This limitation, in an otherwise useful prospective data set, highlights the lack of standardised pain data within an already scarce and conflicting literature pool. This only works to reiterate the importance of being diligent in presenting accurate information to patient's with regard to relative differences in pain, and other oncological and technical factors when considering the type of RP they undergo.

\section{Why is pain important?}

Many factors contribute to deciding the type of RP a patient might undergo at all levels from health-system factors to patient factors. In situations where both RARP and ORP are viable surgical options, it is crucial that surgeons do not misrepresent the purported benefits of one approach compared to another. Whilst intuitively, the more minimally-invasive RARP "should" reduce pain, it is clear that there is no significant difference in post-operative pain between the two approaches. This becomes especially important given the significant bias a patient might have in choosing a surgical approach that would minimise their postoperative pain.

\section{Moving forward/what's next}

Whilst there is no doubt comparative pain between RARP and ORP is one of many metrics that contribute to deciding which operative approach is best for each individual surgeon and patient. It is clear that without a standardised intra- and postoperative analgesics regime and the use of standardised pain score measures, it will be difficult to find consensus in the literature about the little, if any, difference in pain experienced by patients when comparing the two approaches. Moreover, if the purported reduction in pain is being used as part of a rationale to justify RARP over ORP, future studies must endeavour to scrutinies this further. These should be designed by urologists to ensure good uptake and participation with realistic outcomes (16). For example the study presented by Knipper et al. (9) was not 
unblended, leading to potential bias. Also, most urologists that have practiced both approaches know intrinsically there is a difference (for surgeon and patient)—it is just how to accurately measure such differences.

\section{Conclusions}

There is no doubt that there is room clinically for both RARP and ORP to continue to be options for treatment for locally advanced $\mathrm{PCa}$ - each in their own right. In examining the literature further, despite some conflicting evidence, it is clear that benefits of RARP when compared to ORP in a number of metrics, including pain, oncological outcomes and complication rates, are present but in some specific instances may be less than perhaps many thoughts. As always, clinicians should ensure that unsubstantiated claims are not being used to validate clinical decisions, and that we present our patients with realistic expectations of the similarities and differences between the two surgical approaches. We must also accept differences between patients and surgeons and expectations (17) and that only multiple trials accounting for these differences will lead to more confidence in differences between approaches that do exist.

\section{Acknowledgments}

Funding: None.

\section{Footnote}

Conflicts of Interest: The authors have no conflicts of interest to declare.

Ethical Statement: The authors are accountable for all aspects of the work in ensuring that questions related to the accuracy or integrity of any part of the work are appropriately investigated and resolved.

Open Access Statement: This is an Open Access article distributed in accordance with the Creative Commons Attribution-NonCommercial-NoDerivs 4.0 International License (CC BY-NC-ND 4.0), which permits the noncommercial replication and distribution of the article with the strict proviso that no changes or edits are made and the original work is properly cited (including links to both the formal publication through the relevant DOI and the license). See: https://creativecommons.org/licenses/by-nc-nd/4.0/.

\section{References}

1. Ilic D, Evans SM, Allan CA, et al. Laparoscopic and robotassisted vs open radical prostatectomy for the treatment of localized prostate cancer: a Cochrane systematic review. BJU Int 2018;121:845-53.

2. Schiffmann J, Larcher A, Sun M, et al. Differences in Patient Characteristics among Men Choosing Open or Robot-Assisted Radical Prostatectomy in Contemporary Practice - Analysis of Surveillance, Epidemiology, and End Results Database. Urol Int 2017;98:40-8.

3. McCarthy M Jr, Chang CH, Pickard AS, et al. Visual Analog Scales for Assessing Surgical Pain. J Am Coll Surg 2005;201:245-52.

4. Mohiuddin K, Swanson SJ. Maximizing the benefit of minimally invasive surgery. J Surg Oncol 2013;108:315-9.

5. Azhar RA, Bochner B, Catto J, et al. Enhanced Recovery after Urological Surgery: A Contemporary Systematic Review of Outcomes, Key Elements, and Research Needs. Eur Urol 2016;70:176-87.

6. Budäus L, Isbarn H, Schlomm T, et al. Current Technique of Open Intrafascial Nerve-Sparing Retropubic Prostatectomy. Eur Urol 2009;56:317-24.

7. Schlomm T, Tennstedt P, Huxhold C, et al. Neurovascular Structure-adjacent Frozen-section Examination (NeuroSAFE) Increases Nerve-sparing Frequency and Reduces Positive Surgical Margins in Open and Robot-assisted Laparoscopic Radical Prostatectomy: Experience After 11069 Consecutive Patients. Eur Urol 2012;62:333-40.

8. Blazevski A, Scheltema MJ, Amin A, et al. Irreversible electroporation (IRE): a narrative review of the development of IRE from the laboratory to a prostate cancer treatment. BJU Int 2020;125:369-78.

9. Knipper S, Hagedorn M, Sadat-Khonsari M, et al. Comparison of intra- and postoperative analgesia and pain perception in robot-assisted vs. open radical prostatectomy. World J Urol 2019. [Epub ahead of print].

10. D'Alonzo RC, Gan TJ, Moul JW, et al. A retrospective comparison of anesthetic management of robot-assisted laparoscopic radical prostatectomy versus radical retropubic prostatectomy. J Clin Anesth 2009;21:322-8.

11. Yaxley JW, Coughlin GD, Chambers SK, et al. Robotassisted laparoscopic prostatectomy versus open radical retropubic prostatectomy: early outcomes from a randomised controlled phase 3 study. Lancet 2016;388:1057-66.

12. Horowitz M, Wilner N, Alvarez W. Impact of Event 
Scale: A Measure of Subjective Stress. Psychosom Med 1979;41:209-18.

13. Zigmond AS, Snaith RP. The Hospital Anxiety and Depression Scale. Acta Psychiatr Scand 1983;67:361-70.

14. Webster TM, Herrell SD, Chang SS, et al. Robotic assisted laparoscopic radical prostatectomy versus retropubic radical prostatectomy: a prospective assessment of postoperative pain. J Urol 2005;174:912-4; discussion 914.

15. Haese A, Knipper S, Isbarn H, et al. A comparative study

Cite this article as: Condon B, Bagguley D, Lawrentschuk N. Myth busting patient's pain: comparing robotic-assisted verses open radical prostatectomies. Gland Surg 2020;9(2):485-489. doi: $10.21037 /$ gs.2020.01.01 of robot-assisted and open radical prostatectomy in 10 790 men treated by highly trained surgeons for both procedures. BJU Int 2019;123:1031-40.

16. Lawrentschuk N. Urology trial success - get urologists involved early. BJU Int 2019;124 Suppl 1:4.

17. Ranasinghe W, de Silva D, Bandaragoda T, et al. Roboticassisted vs. open radical prostatectomy: A machine learning framework for intelligent analysis of patient-reported outcomes from online cancer support groups. Urol Oncol 2018;36:529.e1-529.e9. 